\title{
Density functional investigations of defect induced mid-gap states in graphane
}

\author{
Bhalchandra S. Pujari, ${ }^{* \dagger}$ and D. G. Kanhere ${ }^{\dagger, \$}$ \\ Department of Physics, University of Pune, Ganeshkhind, Pune-411 007, India., and Centre for \\ Modelling and Simulations, University of Pune, Ganeshkhind, Pune-411 007, India.
}

\footnotetext{
${ }^{\dagger}$ Department of Physics, University of Pune, Ganeshkhind, Pune-411 007, India.

†Centre for Modelling and Simulations, University of Pune, Ganeshkhind, Pune-411 007, India.
} 


\begin{abstract}
We have carried out $a b$ initio electronic structure calculations on graphane (hydrogenated graphene) with single and double vacancy defects. Our analysis of the density of states reveal that such vacancies induce the mid gap states and modify the band gap. The induced states are due to the unpaired electrons on carbon atoms. Interestingly the placement and the number of such states is found to be sensitive to the distance between the vacancies. Furthermore we also found that in most of the cases the vacancies induce a local magnetic moment.
\end{abstract}

\title{
Introduction
}

Graphand i.e. the hydrogenated graphene, is a recent addition to the family of novel carbon materials known for their exotic properties. $\stackrel{1,2,3}{ }$ Recently graphane is experimentally realized by Elias et $a l^{4}$ who demonstrated that the process of hydrogenation is reversible. This observation makes graphane a suitable candidate for the hydrogen-storage material. Equally interesting is the possibility of a direct observation of metal-insulator transition in two dimensional systems. $\frac{\sqrt{5}}{}$ as a function of hydrogen coverage. Graphane is an insulator with a reported theoretical band gap of $\sim 3.5 \mathrm{eV}$ using density functional theory (DFT). 1

It is well known that a single sheet of graphene is susceptible to a variety of disorders like topological defects, impurity states, ripples, cracks etc. Pereira et al ${ }^{6,7}$ have studied different models of local disorders in graphene and have investigated their electronic structure within tight binding method. They have observed a significant changes in the low energy spectrum of graphene viz., localized zero modes, strong resonances, gap and pseudogap behavior etc depending upon the type of disorder. Their results also indicate that by and large disorder significantly modifies the states near the Fermi level. Yazyev and Helm ${ }^{8}$ have studied defect induced magnetism in graphene using DFT. Their work shows that the adsorption of hydrogen or the creation of defect on graphene sheet lead to the local magnetic moment. For an extensive survey of studies of disorders

\footnotetext{
${ }^{1}$ In order to avoid the confusion with the word 'graphene', the word 'graphane' is written in italics throughout this paper.
} 
in graphene we refer the reader to a recent review by Castro Neto et al. $\stackrel{9}{ }$ We wish to point out that most of the novel properties of graphene arise due to the nature of the density of states (DOS) near the Fermi level and these states are sensitive to the presence of defects.

Quite clearly a detailed study of defect induced states is warranted not only for graphene but also for graphane. So far there are no reports of systematic investigations of the effects of such defects on the properties of graphane. In the present work we focus on the electronic structure of graphane with topological defects created by the removal of one and two carbon atoms. Such defects are experimentally realized using high-energy ion beams as demonstrated by Jin et al $\underline{\underline{10}}$ by creating a stable carbon chain from graphene sheet.

The present work is based on spin density functional theory (SDFT), which is known to underestimate the band gap. A recent calculation by Lebègue et al $\underline{11}$ based on GW approximation estimated the band gap of graphane to be $5.4 \mathrm{eV}$. The authors have also shown that the removal of a single hydrogen atom produces mid gap states.

The paper is organized as follows. We present relevant computational details in section ${ }_{\square}$ In section $_{\triangleright}$ the results for pristine graphane is summarized for the purpose of comparison. The main results of the electronic structure calculations on the single and the double vacancy defects are presented in sections ${ }_{\square}$ and ${ }_{\square}$ respectively. Finally the conclusions are presented in section

\section{Computational Details}

All the calculations have been performed on a monolayer graphane, having geometry as described by Sofo et al. $\underline{\underline{1}}$ We have used plane wave based DFT as implemented in Quantum Espresso 2 and VASP $\stackrel{12}{=}$ using the generalized gradient approximation $(\mathrm{GGA}) \stackrel{13}{\underline{13}}, 14$ for exchange-correlation potential. For single vacancy study the primitive graphane cell is repeated 5 times in $X$ and $Y$ directions while for that of double vacancies the repetitions are of 7 units. When creating the vacancy, the carbon and the attached hydrogen are removed. After removing two (four) atoms to form single

\footnotetext{
${ }^{2} \mathrm{http}: / /$ www.quantum-espresso.org/
} 
(double) defect(s) there are total of 98 (192) atoms on the graphane plane. The vertical axis $(Z)$ of the cell is kept as large as $10 \AA$ to avoid the interactions between the graphane sheets. The energy and force thresholds are kept at $10^{-6} \mathrm{eV}$ and $10^{-5} \mathrm{eV} / \mathrm{A}$ respectively. It may be mentioned that even though the unit cell is large it was found to be necessary to use $5 \times 5$ Monkhorst-Pack K-grid for acceptable convergence in energy during the optimization and the self consistency. After optimization we have used $11 \times 11$ Monkhorst-Pack K-grid for final calculations of DOS and other quantities.

\section{Results and discussion}

\section{Pristine Graphane}

Before we present the results of the vacancy studies, it is instructive to summarize the properties of a pure graphane. Our results on pristine graphane are consistent with earlier reports. $\stackrel{1,2}{ }$ Graphane is known to have two distinct conformations depending upon the position of hydrogen atoms with respect to graphene plane. In the chair conformer the hydrogen atoms are attached to carbon atoms in alternating manner to both the sides of the plane while in the boat conformer the pairs of hydrogen atoms are attached in alternating manner. $\frac{1}{-}$ Out of these two the chair conformer is energetically more favorable, hence in the present work we have studied the chair conformer only. It is interesting to note that in graphene the $K$ point of Brillouin zone is degenerate (no gap in DOS) however for graphane the minimum gap is observed at $\Gamma$ point $(\sim 3.5 \mathrm{eV})$ while $K$ point develops rather large gap $\sim 12 \mathrm{eV}$.

We now discuss the DOS of pristine graphane which is shown in Figure Figure 1. The Fermi energy is taken at 0 and is marked by solid vertical line. The largest peak seen (at $\sim-3 \mathrm{eV}$ ) is due to the peculiar $s p^{3}$-like bonding between carbon and hydrogen. The states at the top of the valance band $(\sim-1 \mathrm{eV})$ are mainly comprises of $p$ states forming to the $\sigma$ bonds among the carbon atoms. Unlike graphene there are no $\pi$ bonds in graphane. 


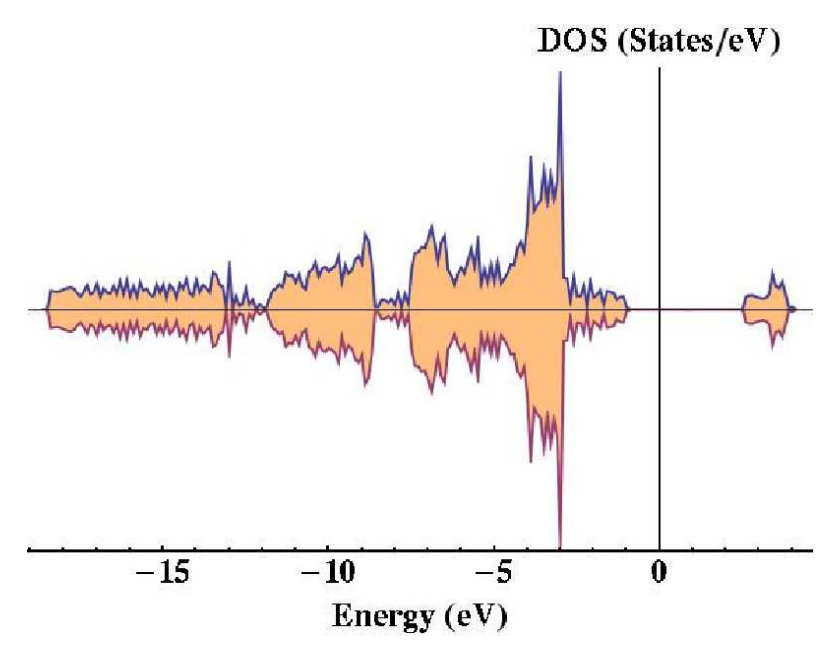

Figure 1: Spin polarized total DOS for pristine graphane. The upper and lower halves of the graph represent up and down spins respectively. The Fermi energy is taken at 0 and marked by a solid vertical line. The system is non-magnetic with a band gap of $\sim 3.5 \mathrm{eV}$.

\section{Single vacancy}

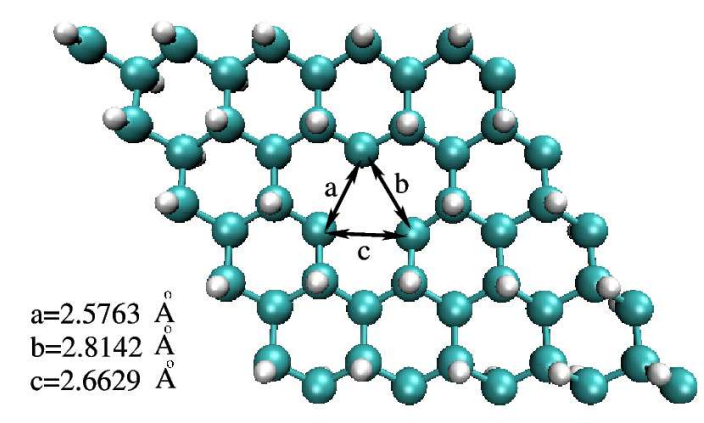

Figure 2: Optimized structure of graphane with a single vacancy. Carbon atoms are shown in cyan color while hydrogen atoms are in white. The distorted nature of the sublattice surrounding the vacancy is clearly evident (marked by the triangle). Pristine sublattice is an equilateral triangle with $a=2.52 \AA$.

We begin our discussion by presenting the study of a single vacancy and its effects on the geometry, electronic structure of graphane. A single vacancy is created by removing one carbon atom along with the attached hydrogen from pure graphane. The fully relaxed structure is shown in figure Figure 2. The immediate effect of the removal of atoms is seen on the sublattice surrounding the vacancy (indicated by the triangle in the figure). In an ideal graphane the carbon sublattice is an equilateral triangle with length of the side $a=2.52 \AA$, however in the presence of vacancy the 
sublattice deforms substantially. Due to reduced coordination number, three carbon atoms on the triangle are pushed away from each other and remain as a part of the hexagon. The distortion of the triangle is asymmetric and the symmetry breaking is due to Jahn-Teller effect. The deformation seen here is qualitatively different than seen in the case of graphene where two of the carbon atoms move close to each other to form a $\sigma$ bond. $\underline{\underline{8}}$ We do not see formation of bonds between the carbon atoms. Thus vacancy leads three dangling bonds on the triangle. As a consequence, the system becomes magnetic with magnetic moment $1 \mu_{B}$. As we shall see these unpaired electrons have an interesting consequences on the DOS.

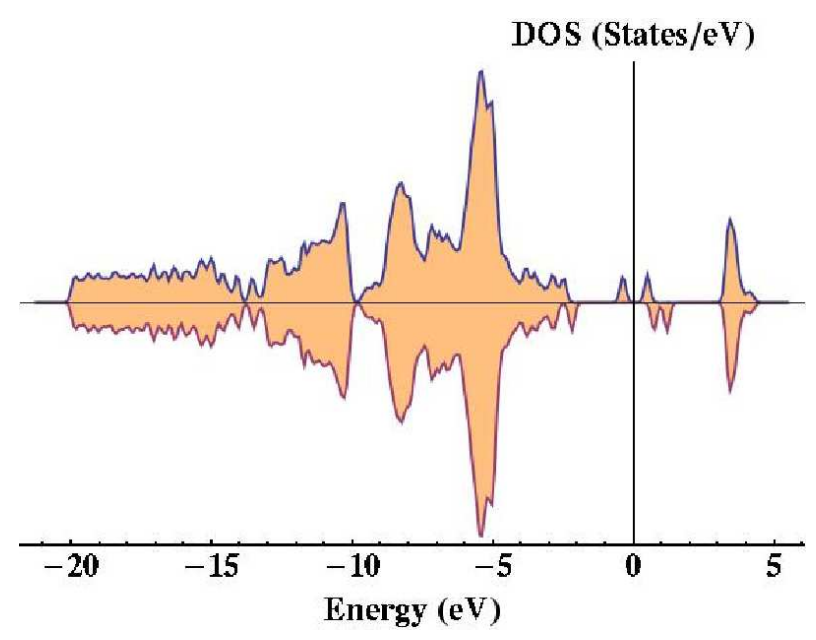

Figure 3: DOS of graphane with single vacancy displaying the induced states in the gap. Fermi energy is at zero and shown by the vertical line. The induced states are partially occupied for up electrons.

Figure Figure 3 shows the spin polarized DOS displaying some remarkable features. The DOS with single vacancy is substantially different from that of pristine graphane ${ }^{1}$ especially near the Fermi energy (marked by a vertical line in the figure). Clearly the vacancy has induced the mid gap states which are partially occupied. In particular the induced spin up states are partially occupied while the induced spin down states are completely empty. The appearance of mid gap states can be attributed to the unpaired electrons from three carbon atoms due to which the states are pushed up from valance band. To ascertain this observation we have hydrogenated three available dangling bonds. Our results show that the increasing hydrogen concentration (from one to three) steadily reduces the density of induced states (figure not shown). Finally with complete hydrogenation, 
induced states vanish and the band gap reduces to $\sim 3 \mathrm{eV}$.

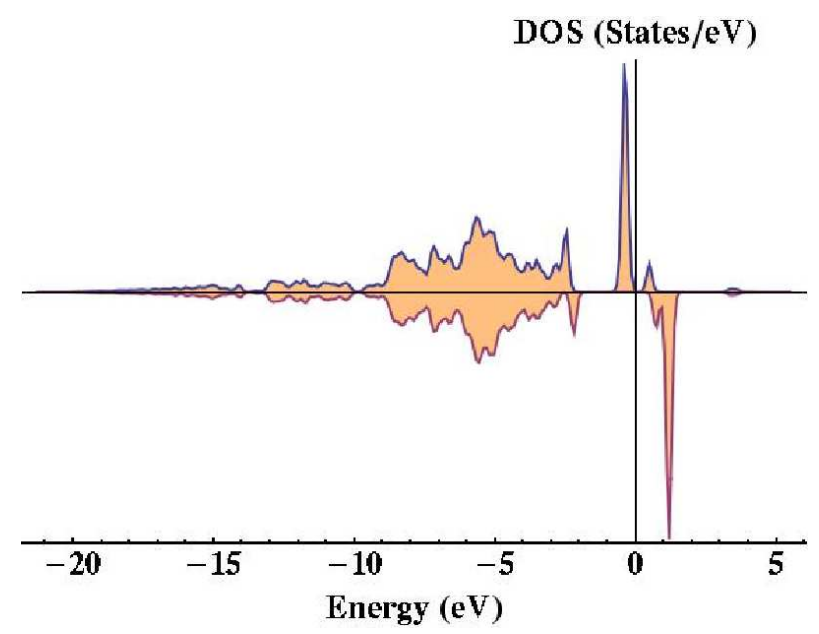

Figure 4: The site projected density of states (PDOS) on one of the carbon atom surrounding the vacancy. It is evident that most of the contribution to the induced states come from the carbon atoms on the sublattice.

That the induced states are due to the unpaired electrons can be further seen from examination of the site projected DOS. Figure Figure 4 shows the site projected DOS (PDOS) on one of the carbon atoms of the triangle. It can be seen that almost all the contribution to the mid gap states come from the three carbon atoms. In fact the contribution from carbon atoms except the three on the sublattice is negligible. Interestingly the hydrogen atoms associated with carbon atom on the triangle do not give any significant contribution. Furthermore the states turned out to be dominantly p-like.

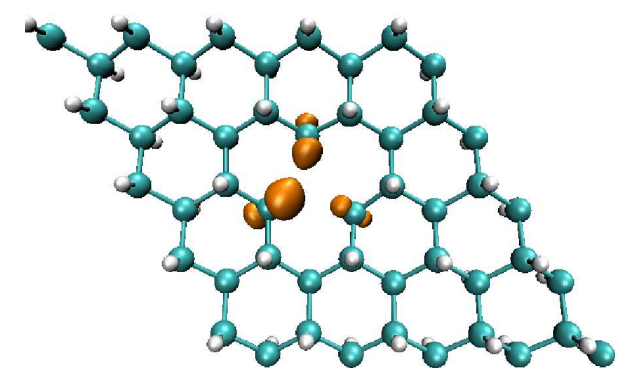

Figure 5: Isosurface of the charge density of the occupied state just below the Fermi level for the case of single vacancy. The state is dominantly $p$-like and is highly localized.

Although there are states near the Fermi energy, these states need not necessarily conduct. Figure Figure 5 shows the isosurface (at one fifth of the maximum value) of the state just below 
Fermi level. This state is induced due to the vacancy and can be seen to be localized. A careful examination of the isosurfaces at the different values reveals that the induced state extends up to three to four nearest neighbouring carbon site. Thus the conduction mechanism through such orbitals may possibly be by hopping.

\section{Double Defect}

The results of a single vacancy gives enough impetus to study the effects of multiple vacancies, especially on the mid gap states. Indeed, as we shall demonstrate, the nature and the placement of induced states are sensitive to the vacancy-vacancy interaction. This aspect brings in an interesting possibility of controlling the effective band gap via creation of vacancy defects.

For the purpose of this study we have used a larger supercell consisting of 192 atoms. Several possible scenarios emerge depending upon the separation of the two vacancies. We have examined four structures obtained by removing 1 ) the closest carbon pair (separation, $D=1.51 \AA$ ), 2) the nearest carbon atoms of the same sublattice $(D=2.52 \AA), 3)$ a pair from different sublattice $(D=$ $3.87 \AA)$ and 4) two carbon atoms having large separation $(D=10.71 \AA)$. In each of the cases we have fully relaxed the structure and have examined the energetics. We have found that the total energy is the lowest when the vacancies are at the closest distance (case 1). The total energies with respect to the energy of the lowest energy system are shown in Table Table 1.

Table 1: Energy difference $\left(\Delta_{E}\right)$ with respect to lowest energy $\operatorname{system}(i . \quad e$. vacancies in vicinity, $D=1.52 \AA$ ). $D$ indicate the separation between the vacancies.

\begin{tabular}{ccccc}
\hline Separation $D$ & $1.51 \AA$ & $2.52 \AA$ & $3.87 \AA$ & $10.71 \AA$ \\
\hline$\Delta_{E}(\mathrm{eV} /$ atom $)$ & 0.0 & 0.0906 & 0.0185 & 0.0338 \\
\hline
\end{tabular}

Figure Figure 6 shows the optimized structure and DOS for the case 1. Remarkably, the carbon atoms surrounding the vacancies rearrange to form two new $\sigma$ bonds leading to the formation of 5-8-5 ringed structure. This peculiar structure does not leave any unpaired electrons (dangling bonds) unlike the case for single vacancy. This is consistent with the observation that there are no mid gap states in the DOS as can be seen from figure Figure 6 (b). In this case the band gap is 


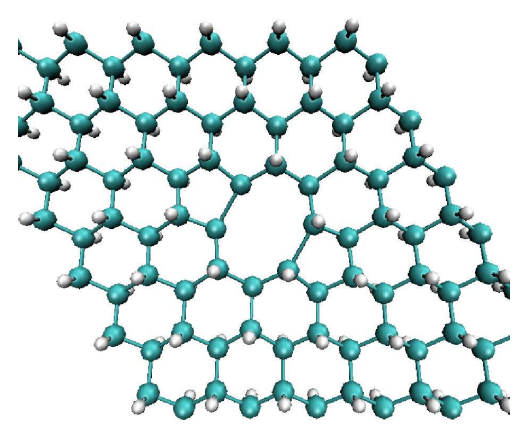

(a)

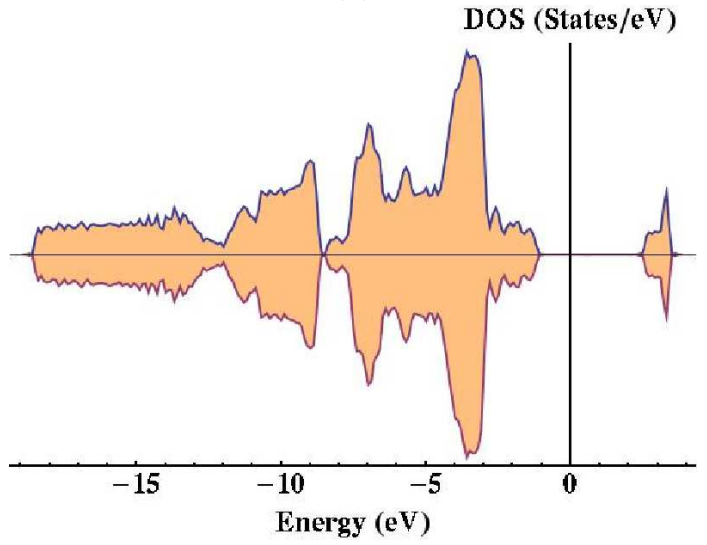

(b)

Figure 6: (a) Optimized structure of graphane with two vacancies for the case 1 (see text). The rearrangement of the carbon atoms around the vacancies can be clearly seen. (b) Spin polarized DOS corresponding to structure in (a). The DOS do not show any induced mid gap states. Due to the rearrangement of the atoms the band gap is reduced by $0.5 \mathrm{eV}$. 
reduced by $0.5 \mathrm{eV}$ with respect to pristine graphane.

In order to ascertain the formation of bonds we have examined the charge densities of relevant states. Figure Figure 7(a) and Figure 7(b) show the charged densities as isosurfaces for two states: one at the top of the valance band and other at the bottom of the conduction band. The isosurfaces are shown at one tenth of their maxima. The charge density of the state in figure Figure 7(a) which corresponds to the valance band (occupied), clearly depicts the formation of $\sigma$ bonds among the carbon atoms. It is interesting to note that the bands at the bottom of the conduction band (figure Figure 7(b)) are highly delocalized with almost no charge around the vacancy site.

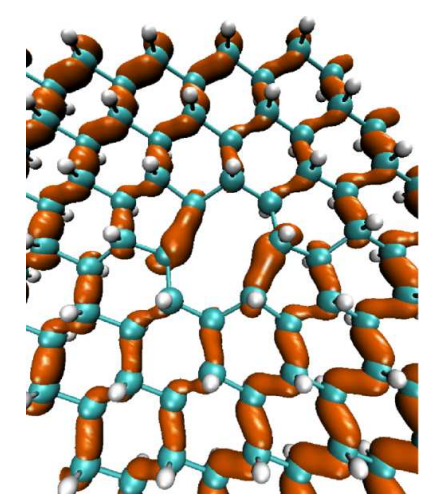

(a)

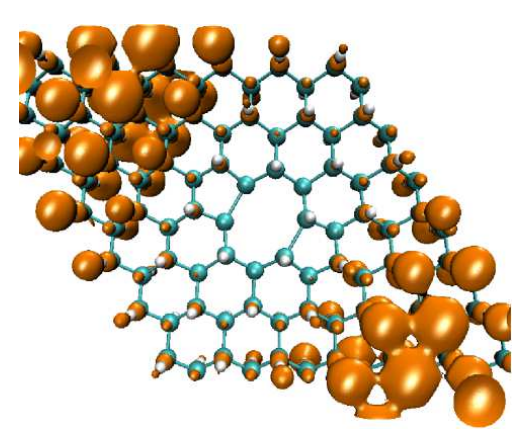

(b)

Figure 7: (a) The charge density of the state at the top of valance band. Two $\sigma$ bonds formed by rearrangement of carbon atoms are clearly seen. (b) Highly delocalized state at the bottom of the conduction band. Both the states resemble those of pristine graphane except for the modifications around the defect site.

A different scenario emerges when the vacancies are separated from each other. Now there are induced states in the gap. Figure Figure 8 shows the DOS for the three cases with the vacancy 


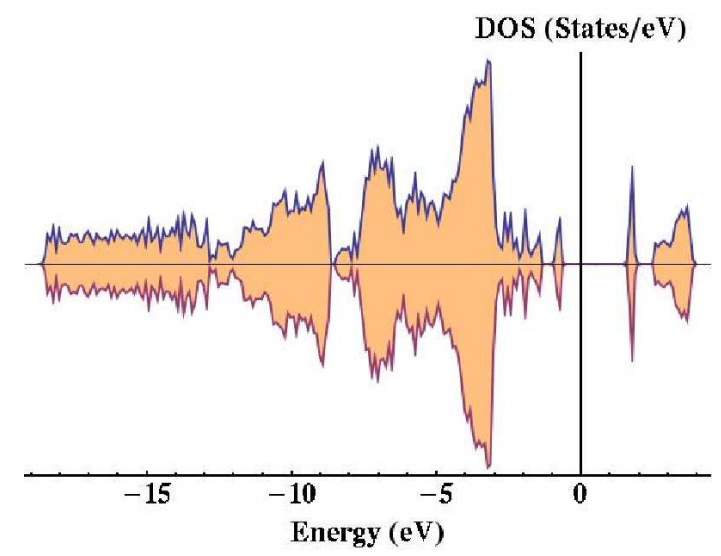

(a): $2.52 \AA$.

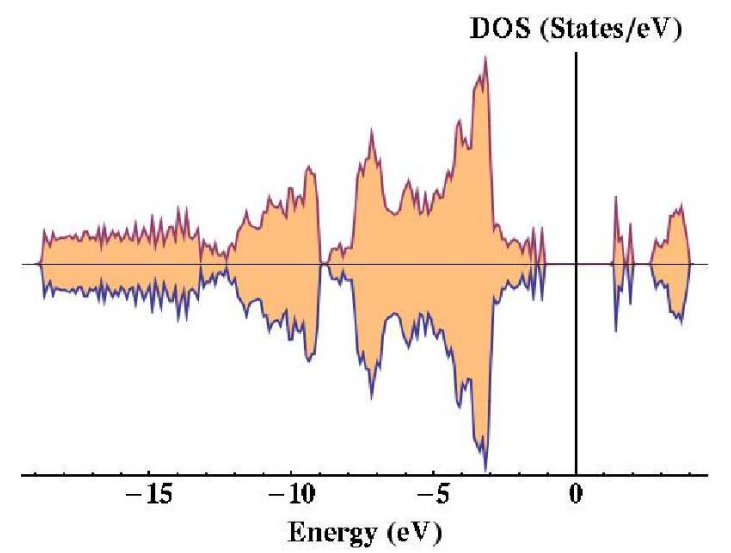

(b): $3.87 \AA$.

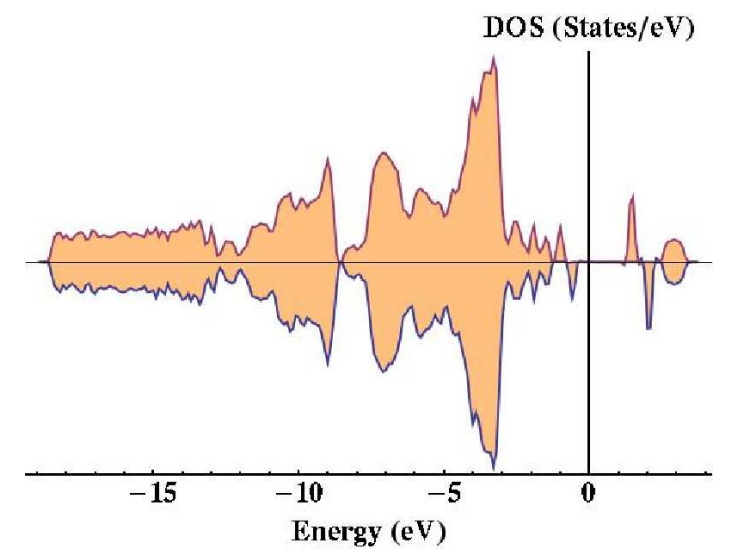

(c): $10.71 \AA$

Figure 8: The DOS of graphane with two vacancies for the cases (2), (3) and (4) (see text). For each of the cases the nature of the induced states are different. (a) The induced states have appeared near the conduction band for the vacancy separation of $2.52 \AA$. (b) For the vacancy separation of $3.87 \AA$ the induced states are broadened, effectively reducing the gap from valance band. (c) A peculiar magnetic system with $2 \mu_{B}$ is seen for the vacancy separation as large as $10.71 \AA$. The induced states are near conduction band and the states on the top of valance band are substantially deformed. 
separation of (a) $2.52 \AA$, (b) $3.87 \AA$ and (c) $10.71 \AA$ respectively. Quite clearly in the case of well separated vacancies, the atoms are not able to rearrange so as to form any bonds. This leaves six unpaired electrons. These unpaired electrons give rise to the mid gap states typically just above the valance band and just below the conduction band. Interestingly their placement is sensitive to the separation between the vacancies. For example, figure Figure 8 (a) shows the DOS for the vacancy separation $D=2.52 \AA$. The induced states are seen at about $0.5 \mathrm{eV}$ below the conduction band along with the additional modifications at the edge of the valance band. As the separation increases to $3.87 \AA$ (figure Figure 8 (b)) the induced states are broadened with a width of about 1 $\mathrm{eV}$ and split in to two distinct peaks. In most of the cases the unpaired electrons tend to cancel the spin giving rise to a non-magnetic states. However for a large separation i. e. in the limit of weakly interacting vacancies, both the vacancies carry a magnetic moment of $1 \mu_{B}$. Figure Figure 8 (c) shows the DOS for system with vacancy separation $10.71 \AA$, depicting the spin polarized features.

The induced states are seen to be localized both in the case of single as well as double vacancies. Figure Figure 9 depicts the charge density isosurfaces corresponding two typical induced states for the case $2(D=3.87 \AA)$. Figure Figure 9 (a) corresponds to the state at the top of the valance band which is occupied while figure Figure 9 (b) corresponds to the unoccupied state below the conduction band. Both the charge densities are plotted at a lower value of isosurfaces (one tenth of their maxima). Clearly the states are dominantly $p$-like and are localized. At this value of isosurface occupied states (figure Figure 9 (a)) is seen to have a weak overlap among the carbon atoms, however at the higher values the lobes are disconnected. On the other hand the charge density of the unoccupied state (figure Figure 9 (b)) is highly localized even at one tenth of the maxima.

Apart from the four cases discussed above, we have also calculated the electronic structure and DOS for a few more vacancy separations. The results indicate that the sublattice plays no role in appearance on mid gap states. 


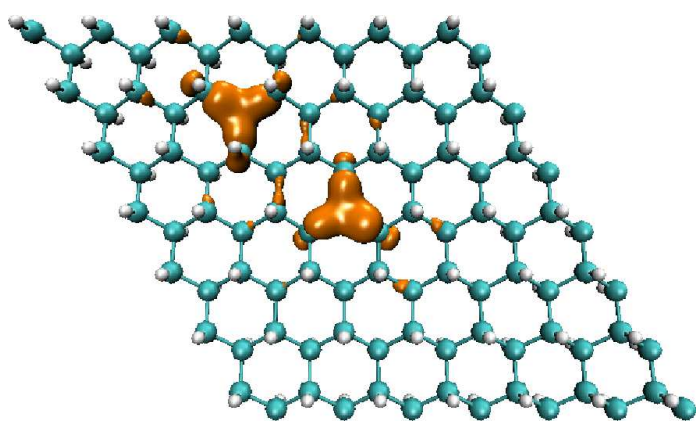

(a)

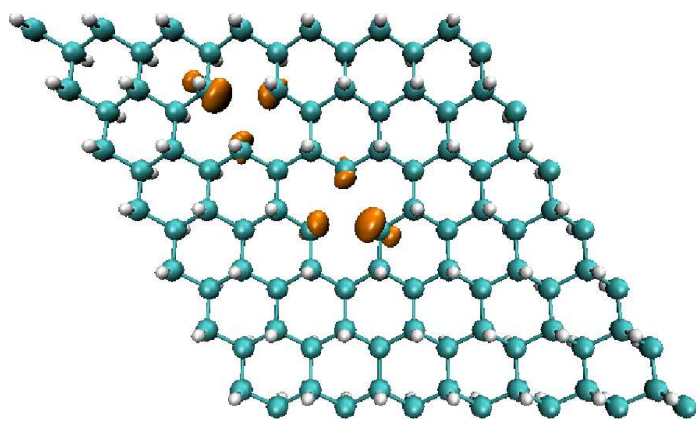

(b)

Figure 9: The charge densities corresponding to innduced states of graphane with two vacancies separated from each other (case 2). (a) The state at the top of the valance band. (b) The state below the conduction band. The isosurface is shown at one tenth of its maximum. 


\section{Conclusions}

We have carried out an ab initio investigation of the electronic structure of graphane with single and double vacancy defects. For both the cases, we have analysed the fully optimized structure, and have examined the DOS and charge densities as a function of separation of the vacancies. Our calculations show that the most stable structure is obtained when the vacancies are adjacent to each other, is accompanied by the reduction of band gap. In this case there are no induced mid gap states. However, separated vacancies induce mid gap states and interestingly their position and width are sensitive to the vacancy separation. Examination of the charge densities of the induced states show that these states are localized. Our calculation brings out the possibility of manipulating the band gap and the nature of the mid gap states with the aid of vacancy defects in graphane.

\section{Acknowledgement}

B.S.P. would like to acknowledge CSIR, Govt. of India for financial support (No: 9/137(0458)/2008EMR-I). It is a pleasure to acknowledge Center for Development of Advanced Computing for computational resources. Some of the figures are generated by using VMD software. 15

\section{References}

(1) Sofo J. O., Chaudhari A. S., Barber G. D. Phys Rev B 2007; 75(15):15340-1-4.

(2) Boukhvalov D. W., Katsnelson M. I., Lichtenstein A. I. Phys Rev B 2008; 77:035427-1-7

(3) Casolo S, Løvvik M, Martinazzo R, Tantardini G. F. J Chem Phys, 2009;130(5):054704-1-10

(4) Elias, D. C., Nair R. R., Mohiuddin T. M. G., Morozov S. V., Blake P., Halsall M. P, et al. Science 2009;323(5914):610-3.

(5) Fuhrer M, Adam S. Nature 2009;458:38-9.

(6) Pereira V M, Loops dos Santos J B M, Castro Neto A H. Phys Rev B 2008; 77:115109-1-17. 
(7) Pereira V M, Guinea F, Loops dos Santos J B M, Peres N M R, Castro Neto A H. Phys Rev Lett 2006; 96:036801-1-4.

(8) Yazyev O V, Helm L, Phys Rev B 2007, 75:125408-1-5.

(9) Castro Neto A H, Guinea F, Peres N M R, Novoselov K S, Geim A K. Rev Mod Phys 2009; 81(1):109-62.

(10) Jin C, Lan H, Peng L, Suenaga K, Iijima S. Phys Rev Lett 2009; 102(20):205501-4.

(11) Lebègue S, Klintenberg M, Eriksson O, Katsnelson M I. Phys Rev B 2009; 79(24):245117$1-5$.

(12) Kresse G, Furthmüller J. Phys Rev B 1996; 54:11169.

(13) Perdew J P, Burke K, Ernzerhof M. Phys Rev Lett 1996; 77:3865-1-4.

(14) Perdew J P, Burke K, Ernzerhof M. Phys Rev Lett 1997; 78:1396.

(15) Humphrey W, Dalke A, Schulten K. J Mol Graphics 1996; 14:33-8. 